\title{
Jesse D. BILLETT, The Divine Office in Anglo-Saxon England (597-c. 1000)
}

Alain Rauwel

\section{(2) OpenEdition}

\section{Journals}

Édition électronique

URL : https://journals.openedition.org/ccm/8182

DOI : $10.4000 / \mathrm{ccm} .8182$

ISSN : 2119-1026

\section{Éditeur}

Centre d'études supérieures de civilisation médiévale/Université de Poitiers

\section{Édition imprimée}

Date de publication : 1 septembre 2021

Pagination : 293-294

ISBN : 978-2-490783-10-6

ISSN : 0007-9731

Référence électronique

Alain Rauwel, « Jesse D. BıLLetT, The Divine Office in Anglo-Saxon England (597-c. 1000) », Cahiers de civilisation médiévale [En ligne], 255 | 2021, mis en ligne le 01 septembre 2021, consulté le 10 février 2022. URL : http://journals.openedition.org/ccm/8182 ; DOI : https://doi.org/10.4000/ccm.8182

\section{(2) $(\mathcal{\theta} \Theta \Theta$}

La revue Cahiers de civilisation médiévale est mise à disposition selon les termes de la Licence Creative Commons Attribution - Pas d'Utilisation Commerciale - Pas de Modification 4.0 International. 


\section{NOTES BRÈVES}

Jesse D. Billett, The Divine Office in Anglo-Saxon England (597-c. 1000), Woodbridge, Boydell Press/ Henry Bradshaw Society (Subsidia, 7), 2019 [1 1 éd. 2014].

Il serait dommage que la somme de Jesse Billett, complexe dans sa construction, profuse dans son information, décourage les historiens de la ritualité et de la vie régulière. Elle affronte en effet de façon très documentée une question importante et étudiée seulement par bribes : l'histoire de la généralisation du cursus bénédictin de l'Office dans les monastères de l'Église latine. Les 463 p. de J. Billett systématisent des aperçus et des intuitions qui n'en occupent au mieux que quelques dizaines dans la synthèse classique de Robert Taft (Robert TAFT, La Liturgie des Heures en Orient et en Occident : origine et sens de l'office divin, Georges PASSELECQ [trad.], Turnhout, Brepols, [Mysteria, 2], 1991). La spécification géographique du titre ne doit pas donner à penser que seuls les spécialistes des mondes insulaires y trouveront leur miel; par un long chapitre introductif intitule « The Divine Office in the Latin West in the Early Middle Ages ", par de nombreuses observations aisément adaptables à d'autres milieux, par des perspectives méthodologiques aussi, l'ouvrage prend en fait valeur de référence sur l'histoire médiévale de la liturgie monastique - y compris en ses précieux tableaux récapitulatifs des structures de l'Office selon les cursus. La très haute qualité scientifique de l'ensemble honore pleinement la tradition de la vénérable Henry Bradshaw Society, éditrice du livre, grâce à laquelle l'Angleterre est un véritable jardin d'Éden des études liturgiques.

L'idée initiale de l'auteur est très juste : la marche vers le monopole de la Règle de saint Benoît chez les moines et moniales d'Occident ne s'est pas accompagnée d'une adoption précoce et générale des prescriptions liturgiques contenues dans ce texte, dont la plus contraignante et la plus visible est la répartition des psaumes, et accessoirement des leçons de l'office nocturne. Le cursus dit « romain » a longtemps primé, y compris dans des communautés parfaitement régulières, et même dans des communautés considérées comme "réformées » selon le type bénédictin. Un bon moyen de le vérifier est l'observation des rubriques dans les psautiers, indiquant généralement les scansions dans la récitation. Le grand moment d'unification des usages lié à Benoît d'Aniane et aux conciles d'Aix a certes fait bouger les lignes, mais moins qu'on ne penserait. Le cursus de la Règle, presque inconnu jusque-là, est resté largement ignoré dans les abbatiales. J. Billett rappelle à juste titre qu'il n'a très vraisemblablement été adopté à Saint-Denis qu'à la fin du $\mathrm{x}^{\mathrm{e}} \mathrm{s}$.

Il serait hautement improbable qu'un mouvement aussi tardif sur le continent ait été précoce en Angleterre : remettre en cause la chronologie défendue par un certain nombre de "classiques » déjà anciens est ainsi l'objet de ce que l'a. présente comme « a new narrative ». Les plus optimistes imaginaient les préceptes de saint Benoît respectés sur l'île dès l'époque d'Augustin de Cantorbéry (ce qui supposerait que la communauté de Grégoire sur le Caelius ait été « bénédictine »!), tandis que d'autres ont postulé que, connus au temps de Bède, ils avaient été noyés sous le flot des invasions et « retrouvés » par les réformateurs du $\mathrm{x}^{\mathrm{e}} \mathrm{s}$. Avec J. Billett, on admet volontiers que l'instauration est bien plus vraisemblable que la restauration, comme il arrive souvent en histoire monastique.

Le $\mathrm{X}^{\mathrm{e}} \mathrm{s}$. prend ainsi dans son récit un relief particulier, marqué qu'il est par les grandes figures de Dunstan, Aethelwold ou Oswald et par le phénomène si caractéristique des îles Britanniques que constituent les cathédrales monastiques. La période antérieure est d'ailleurs très mal connue; avec toute son érudition, J. Billett ne peut consacrer plus d'une quinzaine de pages au siècle qui va de 835 à 940 environ. Dans son analyse, l'a. multiplie les nuances : beaucoup de communautés ont dû être pendant longtemps des corpora permixta, rassemblant des moines stricto sensu et des clerici, avec par conséquent des réticences 
à une bénédictinisation intégrale (qui ne l'était de toute façon jamais puisque la Règle elle-même prévoit à certains jours l'alignement sur le cursus romain). Les schémas généraux ne rendent jamais compte de la pratique liturgique médiévale dans sa diversité, comme l'avait observé Philippe Bernard à propos de la messe, critiquant les rigidités de la classique " théorie des sacramentaires ». Chaque communauté, en fonction de son histoire, de ses moyens, des choix de ses abbés successifs, fixe ses propres normes, toujours en négociation entre des héritages vivement défendus et l'obligation plus ou moins cosmétique de s'adapter aux nouvelles tendances du moment. Reste que les contacts entre les mondes insulaires et le continent se multiplient au milieu $\mathrm{du} \mathrm{X}^{\mathrm{e}} \mathrm{s}$., bousculant les idiosyncrasies. Des chantres venus de Corbie sont présents à Abingdon dans les années 950, et une prestigieuse maison comme Fleury accueille à plusieurs reprises des moines anglais. C'est la Regularis concordia qui exprime pleinement, à défaut de l'achever, le processus de mise en ordre, en valorisant le regularis ordo, désormais bien distingué de la manière de chanter more canonicorum.

Si les textes narratifs ou hagiographiques jouent un grand rôle dans la première moitié de l'ouvrage, les livres liturgiques à proprement parler raflent la vedette dans les deux cents pages suivantes, consacrées à l'analyse de plusieurs manuscrits du $\mathrm{X}^{\mathrm{e}} \mathrm{s}$. On entre ici dans des parages de haute technicité, où les observations codicologiques et l'identification des pièces relèvent de ces jeux de voltige érudite chers à la musicologie médiévale et que les profanes regardent avec le même ébahissement un peu incrédule qu'un spectacle d'acrobates. On aurait tort toutefois de négliger les préliminaires méthodologiques de cette seconde partie, qui donnent sur la méthode des « grands ancêtres », Leroquais et surtout Hesbert, des éclaircissements précieux, pas toujours faciles à trouver, et qui simplifieront la vie aux utilisateurs occasionnels du Corpus antiphonalium officii. Vient alors l'examen minutieux de plusieurs documents. Deux sont connus des spécialistes : le Collectaire de Durham et le ms Cambridge, Corpus Christi, 41, qui renferme la version en vieil anglais de l'Historia ecclesiastica de Bède avec des marginalia liturgiques. Deux autres sont analysés et édités ici par J. Billett : BL, Royal 17.C.XVII et Oxford, Bodleian, Rawlinson D.894. Il est remarquable que l'un comme l'autre soient des fragments. Dans le désert textuel de l'ancienne liturgie anglo-saxonne, c'est par le rassemblement de menues notations éparses que le chercheur peut espérer progresser.

Que notre connaissance de l'histoire liturgique du monde anglo-saxon et de l'Office divin pendant le haut Moyen Âge ait progressé grâce à J. Billett, c'est une certitude. Son enquête, conforme aux exigences scientifiques les plus élevées, tranche heureusement sur de trop nombreuses propositions anglosaxonnes où de micro-rituels peu représentatifs sont montés en épingle au détriment d'une compréhension fondamentale de l'opus Dei. Ici, c'est bien à l'essentiel de l'expérience monastique que s'attaque méthodiquement et fructueusement l'auteur. Certes, les chemins sur lesquels il entraîne son lecteur sont parfois ardus, mais le profit en termes de complexification est certain. Comme le souligne en effet dans son compte rendu de la Catholic Historical Review un orfèvre en la matière, Christopher Jones, le principal mérite de The Divine Office in Anglo-Saxon England est de compliquer notre perception du monachisme ancien. Et compliquer, c'est-à-dire exposer le plus clairement possible la diversité des situations sans les ramener de force à un modèle unique mensonger à force de schématisme, est l'une des marques de fabrique de la bonne histoire.

Alain RAUWEL CeSor-EHESS 